\title{
THE APRIL MEETING IN DURHAM
}

The four hundred forty-fifth meeting of the American Mathematical Society was held at Duke University, Durham, North Carolina, on Friday and Saturday, April 1-2, 1949. The total attendance was about 130 including the following 86 members of the Society:

Louise Adams, D. F. Barrow, W. S. Beckwith, Felix Bernstein, R. G. Blake, T. A. Botts, C. P. Brady, A. T. Brauer, E. T. Browne, G. S. Bruton, R. S. Burington, E. A. Cameron, Leonard Carlitz, M. D. Clement, A. C. Cohen, J. B. Coleman, J. C. Currie, D. C. Dearborn, F. G. Dressel, L. A. Dye, Myrtle Edwards, W. W. Elliott, F. A. Ficken, Tomlinson Fort, A. S. Galbraith, J. R. Garrett, I. C. Gentry, J. J. Gergen, Wallace Givens, P. E. Guenther, O. G. Harrold, I. R. Hershner, Temple Hollcroft, T. R. Hollcroft, Harold Hotelling, G. B. Huff, M. P. Jarnagin, V. L. Klee, J. R. Kline, F. W. Kokomoor, S. H. Lachenbruch, G. B. Lang, J. W. Lasley, C. G. Latimer, M. A. Lee, Marguerite Lehr, Jack Levine, R. J. Levit, P. E. Lewis, E. J. McShane, E. L. Mackie, M. H. Martin, E. P. Miles, B. E. Mitchell, E. E. Moots, C. G. Mumford, J. D. Novak, T. E. Oberbeck, H. V. Park, W. V. Parker, Mary Pettus,' W. T. Puckett, C. N. Reynolds, T. D. Reynolds, R. G. D. Richardson, H. E. Robbins, J. H. Roberts, L. V. Robinson, V. N. Robinson, Clarence Ross, Walter Rudin, H. M. Schwartz, R. E. Smith, W. S. Snyder, H. K. Sohl, R. W. Stokes, B. B. Townsend, H. S. Thurston, L. A. M. Van Dyke, R. Z. Vause, L. I. Wade, J. A. Ward, G. T. Whyburn, W. M. Whyburn, R. L. Wilson, J. W. Young.

Professor J. H. Roberts of Duke University delivered an hour address at 8:30 P.M. on Friday entitled Transformations which change dimension. Professor G. T. Whyburn presided at this session.

Following the invited address a brief informal business meeting was held, Professor W. M. Whyburn presiding. It was agreed that the group would recommend to the Council the acceptance of the invitation of the Oak Ridge National Laboratory to hold the 1950 southeastern meeting in Oak Ridge, Tennessee.

Two sections for contributed papers were held at 9:30 A.M. on Saturday: one in Analysis, Statistics and Applied Mathematics, in which Professor Tomlinson Fort presided, and one in Algebra, Geometry and Topology, in which Professor C. G. Latimer presided.

A dinner for mathematicians and guests was held at 7:00 P.M. on Friday, Professor J. J. Gergen, toastmaster. President A. H. Edens, of Duke University, gave an address of welcome and Professor J. R. Kline spoke regarding the current activities of the Society and the International Congress. A resolution of thanks to Duke University for its hospitality was presented by Professor L. A. Dye and adopted by those present at the dinner.

Abstracts of the papers presented follow below. Abstracts whose numbers are followed by the letter " $t$ " were presented by title. Paper 
number 312 was read by Professor Townsend, number 321 by Professor Robbins. Mr. Gadsden was introduced by Professor T. R. Hollcroft, Mr. Peters by Professor Tomlinson Fort, Mr. Blumen and Dr. Nicholson by Professor H. E. Robbins, and Mr. Keesee by Professor A. D. Wallace.

\section{Algebra and Theory of Numbers}

\section{A. T. Brauer: Limits for the roots of algebraic equations.}

The results of the papers Limits for the characteristic roots of a matrix I, II, III, Duke Math. J. vol. 13 (1946) pp. 387-395, vol. 14 (1947) pp. 21-26, vol. 15 (1948) pp. 871-877, are used to prove well known theorems on the location of the roots of algebraic equations in a unified way while the original proofs are very different. Moreover, most of the results obtained in this paper give better bounds for the roots than the original theorems. For instance, it is proved that all the zeros of $f(x)=x^{n}+a_{1} x^{n-1}$ $+\cdots+a_{n}$ lie in the closed unit circle or in the oval of Cassini $\left|z\left(z+a_{1}\right) \leqq\right| a_{2}|+| a_{3} \mid$ $+\cdots+\left|a_{n}\right| \cdot$ (Received February 17, 1949.)

\section{Leonard Carlitz: Hurwitz series: Eisenstein criterion.}

By an $H$-series is meant one of the form $\sum_{0}^{\infty} c_{m} x^{m} / m$ !, where the $c_{m}$ are rational integers. (For some previous results see Duke Math. J. vol. 8 (1941) pp. 689-700.) The $H$-series evidently form a domain of integrity; factorization is not in general unique. The principal result of the present paper is an analogue of the Eisenstein criterion for series with rational $c_{m}$, that is, a necessary condition that such a series satisfy an equation $\sum_{0}^{r} A_{i} w^{i}=0$, where the $A_{i}$ denote $H$-series. (Received February 17, 1949.)

\section{4t. Leonard Carlitz: Some properties of Hurwitz series.}

Let $f(x)=\sum_{1}^{\infty} c_{m} x^{m} / m !, c_{1}=1, c_{m}$ integral, and assume that the inverse function is of the form $\lambda(x)=\sum_{1}^{\infty} \epsilon_{m} x^{m} / m, \epsilon_{m}$ integral (see Duke Math. J. vol. 8 (1941) pp. 689700). Among the results of this paper are: (1) $c_{m+p-1} \equiv c_{m}(\bmod p)$; a like result holds for powers of $f(x) ;(2) x / f(x)$ has integral coefficients if and only if $x / \lambda(x)$ has integral coefficients. A number of results on the coefficients of the reciprocal of an arbitrary Hurwitz series are also derived. (Received February 23, 1949.)

285t. Graham Higman, B. H. Neumann, and Hanna Neumann: Embedding theorems for groups. Preliminary report.

The following theorem is proved: Two subgroups $A$ and $B$ of a group $G$ are conjugate in a suitable group $H$ containing $G$ as a subgroup if and (trivially) only if they are isomorphic; and then every isomorphic mapping of $A$ onto $B$ can be obtained by a transformation in a suitable $H$. Using this theorem one can construct infinite groups in which all elements except the unit element are conjugate to each other. Another consequence is the theorem that every countable group $G$ is a subgroup of a group $H$ with two generators. If $G$ can be defined by $n$ defining relations, then one can define the two-generator group $H$ also by $n$ relations. (Received February 17, 1949.)

286. R. J. Levit: A criterion for the irreducibility of polynomials in the rational field. 
Several tests for the irreducibility of a polynomial in the field of rationals are known which depend upon the values assumed by the polynomial on a finite set of integers. G. P6lya proved (Jber. Deutschen Math. Verein. vol. 28 (1919) pp. 31-40) the following: Let $P(x)$ be an integral polynomial of degree $n$. If a set of $n$ integers $x_{1}, x_{2}, \cdots, x_{n}$ can be found such that $0<\left|P\left(x_{i}\right)\right|<M, i=1,2, \cdots, n$, where $M$ is a certain number depending only on $n$, then $P(x)$ is irreducible in the field of rationals. Later writers have sharpened this test by obtaining higher values for the bound $M$, the highest previously known being due to A. T. Brauer and G. Ehrlich (Bull. Amer. Math. Soc. vol. 52 (1946) pp. 844-856). In the present paper a still larger bound is obtained, namely, $M=a(a+1)(a+2) \cdots(a+b) / 2^{b}$, where $a=2^{-1}[n / 2]$ and $b=[(n-1) / 2]$. The proof depends upon the properties of a family of polynomials which are a generalization of the Tchebycheff polynomials. (Received February 17, 1949.)

\section{W. V. Parker: On the matrix equation $A X=X B$.}

A method is given for writing the matrix $X$ such that $A X=X B$ in terms of parameters when $A$ is in rational canonical form. Let $S$ be the set of all matrices commutative with $A$ and let $k$ be the number of invariant factors of $\lambda I-A$. Associated with every matrix $X$ of $S$ is a matrix $M(\lambda)$ whose order is $k$ and whose elements are polynomials in $\lambda$. If $F(\mu, \lambda)=\left|\mu I_{k}-M(\lambda)\right|$ then $F(X, A)=0$. This reduces to the Hamilton-Cayley theorem for $k=n$ and to $X=M(A)$ for $k=1$. (Received February 11, 1949.)

\section{8t. K. G. Ramanathan: On certain identical relations between modular functions.}

If $f_{\nu}(\tau)=T\left[\left(\eta\left(p^{2} \tau\right) / \eta(\tau)\right)^{\nu}\right]$ where $\eta(\tau)$ is Dedekind's modular form and $T$ is Hecke's linear operator (Math. Ann. vol. 117, p. 1) it is shown that between any $p+1$ consecutive $f_{\nu}(\tau)$ there is a linear relation, with coefficients which are independent of $\nu$. In the cases $p=5,7,13$ this linear relation with $\nu=1, \cdots, p+1$ is exactly the irrational modular equation connecting $\eta\left(p^{2} \tau\right) / \eta(p \tau)$ and $\eta(p \tau) / \eta(\tau)$. When $p=11$ it is shown that the linear relation could be used in an attack on Ramanujan's problem of the congruences of the partition function. (Received February 16, 1949.)

\section{9t. Walter Rudin: A theorem on Hurwitz series.}

By an $H$-series of type $k(k \geqq 0)$ is meant a formal power series $f(x)=\sum a_{m} x^{m} /\{m, k\}$ where the coefficients $a_{m}$ are rational integers, and $\{m, k\}=m !(m+1) ! \cdots(m+k) ! / 1$ ! $\cdots k !,\{m, 0\}=m !$. For a fixed $k$, the $H$-series of type $k$ form a domain of integrity, denoted by $H_{k}$. The following theorem is proved. Let $P$ be an infinite set of distinct rational primes. Let $f(x) \in H_{k+1}$, and suppose that $a_{p} \not \equiv 0(\bmod p)$ for $p \in P$. Then $f(x)$ is transcendental relative to $H_{k}$. (Received March 24, 1949.)

\section{L. I. Wade: Multiply periodic functions over $G F[p, x]$.}

Using the valuation of $G F(p, x)$ determined by the degree in $x$, let $\mathfrak{M}$ denote the algebraically closed, complete field containing this topological field. The interest is in certain entire functions, that is, power series in $t$ over $\mathfrak{M}$ that converge for all elements of $M$. Such a function $f(t)$ is called periodic over $G F[p, x]$ with period $\omega$ if $f(t+A \omega)$ $=f(t)$ for $A \in G F[p, x]$. A necessary and sufficient condition that an entire function exist with the periods $\omega_{1}, \cdots, \omega_{n}$, linearly independent over $G F(p, x)$, is that $\omega_{1}, \cdots, \omega_{n}$ be linearly independent over the completion of $G F(p, x)$. The function $f(t)$ with the zeros $A_{1} \omega_{1}+\cdots+A_{n} \omega_{n}, A_{i} \in G F[p, x]$, and no other zeros, is a linear 
function (of the form $\sum \alpha_{i} t^{i}$ ); and, furthermore, has the multiplication theorem $f(M t)=\sum_{0}^{n} \gamma_{i} f^{i}(t), \gamma_{i} \in \mathfrak{M}$. Conversely, this relation determines an $n$-ply periodic function of this type. Similar theorems are proved about entire functions with infinitely many periods (in particular, their existence). Also, multiple periodicity over $G F\left[p ; x_{1}, \cdots, x_{n}\right]$ is treated. (Received February $\left.18,1949.\right)$

\section{J. A. Ward: Solution of generalized Cauchy-Riemann differen-} tial equations by linear algebras.

The author proves the following theorem: If $A_{i}=\left(a_{i s r}\right)(i=1,2, \cdots, n)$ is a set of linearly independent $n$ by $n$ matrices over a field $F$ such that $a_{i 1 r}=\delta_{i r}$ and $A_{i} A_{j}$ $=A_{j} A_{i}$ for all $i$ and $j$, then $A_{i} A_{j}=\sum_{k=1}^{n} a_{i j k} A_{k}$. This theorem is used to establish necessary and sufficient conditions that the system $\sum_{i, j-1}^{n} d_{i j k} \partial y_{i} / \partial x_{j}=0$, $k=1,2, \cdots,\left(n^{2}-n\right)$, where the $d_{i j k}$ are constants in $F$, shall be the generalized Cauchy Riemann differential equations of a linear, associative, commutative algebra $A$ of order $n$ with an identity element over $F$. (See J. A. Ward, $A$ theory of analytic functions in linear associative algebras, Duke Math. J. vol. 7 (1940) pp. 233-248.) If the conditions are satisfied the author is able to determine the algebra $A$ and give the solutions of the differential equations as a power series in the algebra. (Received February 14, 1949.)

\section{ANALysis}

292. Felix Bernstein: A new integral relation of the theta zero function from which its natural boundary can be obtained.

The characteristic integral equation of the Volterra type of the theta zero function derived by the author in 1926 in order to solve a problem in heat conduction enables one to establish all the known relations of the theta zero function to problems of the conduction of heat. An integral relation which is a generalization of this integral equation leads to a simple proof of the existence of a natural boundary for the theta zero function. This is a new approach to the gap theorems and to the study of the behavior of an analytic function in the neighborhood of its natural boundary. (Received April 2, 1949.)

293. C. P. Gadsden: On the zeros of the solutions of systems of homogeneous linear differential equations.

The system considered is (1) $x^{\prime}=A(t) x$, where $x=\left[x_{i}(t)\right]$ is a column matrix and $A(t)=\left[a_{i j}(t)\right]$ is an $n \times n$ matrix of class $C^{m}$ on $R$ : $-\infty<t<\infty$, for some $m=0,1, \cdots, \infty$. For a solution $x \neq 0$ of (1) define the set $X_{i}=x_{i}^{-1}[0]$ of zeros of $x_{i}(t)$. Then (i) $X_{i}$ is closed and (ii) $\bigcap X_{i}=0$. The conditions (i) and (ii) are also sufficient, in the following sense. Let $\left[X_{i}\right]$ be $n$ subsets of $R$ satisfying (i) and (ii). Then there exist matrices $A(t)$ and $x$ of class $C^{\infty}$ satisfying (1) and such that $x_{i}^{-1}[0]=X_{i}$. The proof depends on the fact that, for any closed set $Z \subset R$, a function $f(t)$ of class $C^{\infty}$ on $R$ can be defined so that $f^{-1}[0]=Z$. Similar results are given for $A(t)$ analytic. For the case $n=2$, some separation theorems are given, assuming that one of the conditions $\pm A_{i j}(t) \geqq 0, i \neq j$, holds. (Received February 16, 1949.)

\section{4t. V. L. Klee: $A$ note on 8-functionals.}

Let $R$ denote the real number field and $R^{+}=R \cup\{\infty\}$. A $\wp-\left(\left(\wp^{+}-\right)\right)$functional on a real linear system $L$ is a positively homogeneous subadditive function on $L$ to 
$R\left(\left(R^{+}\right)\right)$. The following results are established: (1) If $p$ is a $\wp^{+}$functional and $-q$ is a $\varnothing$-functional such that $q \leqq p$, then there is a linear functional $f$ on $L$ such that $q \leqq f \leqq p$; (2) If $p_{1}$ and $p_{2}$ are $\wp$-functionals and $f$ is a linear functional such that $f \leqq \sup \left(p_{1}, p_{2}\right)$, then there are linear functionals $f_{i} \leqq p_{i}$ and $a \in[0,1]$ such that $f=a f_{1}+(1-a) f_{2}$. By means of these a new proof is given for some results of Krein and Smulian (Ann. of Math. vol. 41 (1940) pp. 561-564, 567) on regularly convex sets. (Received February 23, 1949.)

\section{5t. V. L. Klee: $A$ second note on 8 -functionals.}

(For the definition of $\wp$ - and $\wp^{+}$-functionals see the preceding abstract.) A new proof of the Hahn-Banach extension theorem (concerning $\wp$-functionals on an arbitrary linear system) is given. On finite-dimensional systems there is obtained a simple characterization of those $\wp^{+}$-functionals for which the Hahn-Banach theorem remains valid, and it is shown that each $\wp^{+}$-functional majorizes at least one linear functional. (Received February 23, 1949.)

\section{6t. V. L. Klee: On dense convex sets.}

Let $E$ be an infinite-dimensional normed linear space. It is shown that for subspaces $S$ of $E$ the property of having a dense complementary subspace depends only on the deficiency of $S$. In particular, $E$ must have a dense proper subspace. From this it is shown that if $D$ is the affine dimension and $N$ the cardinal number of $E$, and either $2 \leqq M \leqq D$ or $M=N$, then $E$ can be expressed as the union of $M$ pairwise disjoint dense convex sets. This strengthens a result of J. W. Tukey (Portugaliae Mathematica vol. 3 (1942) p. 101). (Received February 23, 1949.)

\section{7t. V. L. Klee: On a conjecture of Bourgin.}

A subset $X$ of a Hausdorff linear space $H$ is called "regularly $H$ convex" if to each $y \in H-X$ corresponds a continuous linear functional $f$ on $H$ such that $f(y)$ $>\sup _{x} \in X f(x)$. D. G. Bourgin (Amer. J. Math. vol. 65 (1943) pp. 637-659) has shown that $(\alpha) H$ is locally convex implies $(\beta)$ every closed convex subset of $H$ is regularly $H$ convex. The present paper the author describes a class of Hausdorff linear spaces for which $(\beta)$ is true but $(\alpha)$ is not, thus substantiating a conjecture of Bourgin. (Received February 23, 1949.)

\section{V. L. Klee: On convex sets in linear spaces.}

A $(T \alpha)$-space $E$ is at once a real linear system and a topological space, the two structures being so related that for each $y \in E$ and real $r$, the function $y+r x \mid x \in E$ is continuous. It is shown that many of the known properties of convex sets in normed linear spaces are still present in $(T \alpha)$-spaces, and of those which fail in some $(T \alpha)$ spaces several important ones are valid at least in every locally convex Hausdorff linear space. A fundamental result is the following: If $A$ and $B$ are disjoint convex subsets of the real linear system $L$ and one of them has a radially interior point, there is a linear functional $f \neq 0$ on $L$ and a constant $c$ such that $f \geqq c$ on $A$ and $f \leqq c$ on $B$. Among the topics treated are radial interiority, existence of continuous linear functionals, and support, separation, and non-support properties of convex sets. The principal theorems extend to less restricted spaces results of D. G. Bourgin, J. P. LaSalle, S. Mazur, J. W. Tukey, and the author. (Received February 23, 1949.)

299t. N. Metropolis and S. M. Ulam: On motions of systems of mass points randomly distributed on the infinite line. 
Assume that $x=. \alpha_{1} \alpha_{2} \cdots \alpha_{n} \cdots$ is a normal number (in the sense of Borel) in the binary system corresponding to a system of mass points, each of mass 1 located on the infinite line; or the coordinate $i$ if $\alpha_{2 i}=1$, or $-i$ if $\alpha_{2 i+1}=1$. This distribution corresponds to time $t=0$. The points attract each other with forces inversely proportional to the squares of their respective distances. A study is made of asymptotic properties of the motion of such systems. In particular, one proves the existence of condensations and super-condensations of various orders. (Received February 18, 1949.)

300t. Paolo Nesbeda: On the minimum number of self-intersections of a locally simple closed curve. Preliminary report.

Let $g$ be a L-S-closed curve intersecting itself in a finite number of points, and let $[g]$ denote the 0 -homotopy class of $g$. A curve of $g$ which is admissibly deformable into a curve $g^{\prime}$ without altering the self-intersections and so that on a sufficiently small circle with center at an arbitrary self-intersection $Q$ of $g^{\prime}$ the four subarcs of $g^{\prime}$ with end point $Q$ are straight is defined as a curve of model intersections. If the model self-intersections are defined to be of type $A$ if the incoming straight arcs are adjacent, it is shown that the intersections of $g$ if model and minimum in number are necessarily of type A. It is shown further that the minimum number of self-intersections is $N=|q|$ $-|p-q|-1$ where $p$ and $q$ denote the angular order and the order of $g$ with respect to 0 , respectively. The proof is combinatorial in character. Some of the results and methods used are taken from the work of M. Morse, Topological methods in the theory of functions of a complex variable. (Received February 18, 1949.)

301. G. O. Peters: Bernoulli polynomials and numbers of the second kind of higher order and of negative order. Preliminary report.

Nörlund, in his Vorlesungen ïber Differenzenrechnung, defines Bernoulli polynomials and numbers of higher order and of negative order. Jordan, in his Calculus of finite differences, defines Bernoulli polynomials and numbers of the second kind, but does not treat the higher orders or negative orders of these polynomials and numbers. The author denotes Bernoulli polynomials and numbers of the second kind, of order $n$ and degree $v$, by $b_{v}^{n}(x)$ and $b_{v}^{n}$, respectively, defined by: $b_{v}^{0}(x)=x^{(v)}=x(x-1)(x-2) \cdots$ $(x-v+1) ; \quad b_{v}^{n}(x)=\int_{0}^{x} v b_{v-1}^{n-1}(x) d x+b_{v}^{n} ; \quad b_{v}^{n}(x)=\sum_{i=0}^{0} v v_{i} b_{i}^{n} x^{(v-i)}=\left(x+b^{n}\right)^{(v)} ; \quad v=0,1$, $2, \cdots ; n=0, \pm 1, \pm 2, \cdots$. Properties and applications of these polynomials are found; one of which allows integration (or differentiation) of certain polynomials, which would be tedious by ordinary means, to be performed by summation (or differencing), which becomes simple for these cases. Formulas, involving Sterling's numbers of the second kind, are found for evaluating the Bernoulli numbers of the second kind of higher order. The symmetry of the polynomials is found to be given by $b_{v}^{n}(x)=(-1)^{v} b_{v}^{n}(v-n-1-x)$. (Received February 18, 1949.)

302. L. V. Robinson: Intermediate integrals of nonlinear second order partial differential equations by operational methods.

By means of a differential operator, it is shown how an intermediate integral of a nonlinear second order partial differential equation can be easily obtained. (Received April 1, 1949.)

\section{Walter Rudin: Uniqueness theory for Laplace series.}

Let $P$ denote a point on $S$, the surface of the unit sphere. Let $Y_{n}(P)$ be a spherical 
surface harmonic of degree $n$. Put $\Lambda F(P)=\lim _{h \rightarrow 0}(m(F ; P, h)-F(P)) / \sin ^{2}(h / 2)$, where $m(F ; P, h)$ denotes the mean of $F$ on the circle of intersection of $S$ and the sphere of radius $2 \sin (h / 2)$ about $P$. Define $\Lambda^{*} F(P), \Lambda_{*} F(P)$ similarly, with lim sup, $\lim$ inf in place of $\lim$. The series $\Sigma=\sum_{0}^{\infty} Y_{n}(P) \in K$ if $-\sum_{1}^{\infty} Y_{n}(P) / n(n+1)$ is the Laplace series of a function $F(P)$ continuous on $S$. Let $Z$ be a closed set of capacity zero on $\mathrm{S}$. The following two theorems are the main result of the paper. I. Let $\Sigma \in K$. Suppose (a) $\Lambda^{*} F(P)>-\infty, \Lambda_{*} F(P)<+\infty$ on $S-Z$; (b) there exists $y \in L$ on $S$ such that $\Lambda^{*} F(P) \geqq y(P)$ on $S$. Then $\Lambda F(P)$ exists p.p. on $S$, and $\Sigma$ is the Laplace series of $Y_{0}+\Lambda F(P)$. II. Let $\Sigma \in K$. Suppose (a) the upper and lower Poisson sums ( $f^{*}$ and $f_{*}$ ) of $\Sigma$ are finite on $S-Z$; (b) there exists $y \in L$ on $S$ such that $f_{*}(P) \geqq y(P)$ on $S$. Then $\Sigma$ is Poisson summable p.p. on $S$, and is the Laplace series of its Poisson sum. (Received February 9, 1949.)

\section{H. M. Schwartz: Contributions to existence theory of ordinary differential equations in the real domain. II.}

In part I (Bull. Amer. Math. Soc. Abstract 55-5-521) were discussed conditions that are sufficient for the existence, unique or otherwise, of a solution of a system of differential equations $y_{i}^{\prime}(x)=f_{i}\left(x, y_{1}, \cdots, y_{n}\right)(i=1,2, \cdots, n)$ with preassigned value at a given point of the interval of definition I. In the present paper that discussion is extended in two directions. The requirement of finiteness of the in terval $I$ is removed, and the conditions of existence of solutions of the required type are shown to be the best in a certain specified sense. The extension of the results to the infinite interval is based on the following lemma (for related results in the finite interval cf. Caratheodory, Reelle Funktionen, I, 1939, pp. 172-180): a sequence of functions uniformly bounded and equicontinuous in an interval $I$, finite or infinite, contains a subsequence uniformly convergent in $I$. (Received February 17,1949.)

305. W. S. Snyder: Regular points of families of sets. Preliminary report.

Consider families $F$ of closed subsets of a bounded open set $G$ in Euclidean $N$-space. A point $x$ is an $\alpha$-regular point for $F$ if there is a subfamily of disjoint sets $F_{1} \subset F$ such that the union $x+\sum x \in F_{1} X$ has parameter of regularity (Saks, Theory of the integral, 1937, p. 106) greater than $\alpha$. Let $R(F, \alpha)$ denote the set of points which are $\alpha$-regular for $F$, and $R(F)=\sum_{n=2}^{\infty} R(F, 1 / n)$ is termed the set of regular points for $F$. The theorem is proved: If the sequence of families $\left\{F_{n}\right\}_{1}^{\infty}$ satisfies the conditions (1) each $F_{n}$ is a finite family of disjoint, closed subsets of $G$, (2) the norm of $F_{n}$ converges to 0 as $n \rightarrow \infty,(3)\left|\sum_{x \in F_{n}} x\right| \geqq \beta>0$, then $\left|\sum_{j=1}^{\infty}\left\{\lim \sup _{n} R\left(F_{n}, 1 / j\right)\right\}\right| \geqq \beta$ and $0<\gamma<1$ implies that $\left|R\left(\sum_{n=1}^{\infty} F_{n}, \gamma\right)\right| \geqq \beta$. In the proof, the author uses standard methods of Lebesgue theory. The interest of the theorem is due to its being effectively a converse of the Vitali covering theorem. It can be used to prove that certain derivatives (R. C. Young, Functions of $\Sigma$, and so on, Math. Ann. vol. 29 (1928)) are essentially independent of the parameter of regularity involved in their definitions. (Received February 23, 1949.)

306. W. M. Whyburn: Existence and oscillation theorems for second order, nonlinear, differential systems.

The differential system $y^{\prime}=K(x, y, z ; \lambda) z, z^{\prime}=G(x, y, z ; \lambda) y$, together with two linear boundary conditions at two points, is considered. Under mild hypotheses on the coefficients of the equations and boundary conditions, the existence of sets of characteristic numbers for the system is established. Oscillation theorems are given for the 
characteristic solutions and other properties of these solutions are demonstrated. In the present paper, results previously obtained by the author (Trans. Amer. Math. Soc. vol. 30 (1928) pp. 630-640, 848-854) are extended. The methods used are similar to those of the earlier paper. (Received February 14,1949.)

\section{7t. Bertram Yood: Banach algebras of continuous functions.}

Let $X$ be a bicompact Hausdorff space and $C=C(X, B)$ be the Banach algebra of all continuous functions $f(x)$ with domain $X$ and values in a commutative Banach algebra $B$ with a unit. The norm $\|f\|$ is given by l.u.b. $\|f(x)\|, x \in X$. If the maximal ideals of $C$ are all of the form, the set of all $f$ in $C$ such that $f\left(x_{0}\right) \in M$, where $x_{0} \in X$ and $M$ is a maximal ideal of $B$; then the space of maximal ideals of $C$ is homeomorphic to the Cartesian product $X \times \mathfrak{M}$ where $\mathfrak{M}$ is the space of maximal ideals of $B$. The hypothesis of this theorem is verified if $B$ is a ${ }^{*}$-algebra in the sense of Arens (Duke Math. J. vol. 14 (1947) pp. 269-282). Other aspects of the ideal theory of $C$ are also considered. (Received February 18, 1949.)

\section{Applied Mathematics}

308t. R. A. Clark, Eric Reissner, and G. B. Thomas: On the theory of generalised plane stress.

The authors are concerned with a problem of three-dimensional elasticity for bodies bounded by cylindrical surfaces and by two parallel planes at right angles to the cylindrical surfaces. It is assumed that the plane portions of the surface are free of stress and that the stresses applied to the cylindrical surfaces do not vary along the generators of these surfaces. The object of the study is the determination of threedimensional corrections for the two-dimensional theories of plane stress and plane strain. For this purpose the direct methods of the calculus of variations are used to obtain a system of approximate two-dimensional partial differential equations for the three-dimensional problem. The general equations obtained are used for the solution of a specific problem. (Received February 23, 1949.)

\section{P. E. Guenther: Numerical solution of panel heat flow prob- lems.}

The two-dimensional problem describing the steady flow of heat in a panel from sources embedded in a panel to the panel surfaces is complicated by nonlinear boundary conditions imposed by radiative and convective transfer conditions at the panel surfaces. Temperature distributions within the panel for a number of cases involving typical operating conditions are determined by relaxation methods. The panel surfacetemperature distributions so obtained and the rates of heat flow across the panel surfaces computed therefrom are compared with observed values of the same quantities determined from operation of a full-scale model panel. The effect of the refinement of net size upon improving the agreement between computed and observed results at the expense of complicating and extending the calculations is investigated. (Received February 23, 1949.)

\section{M. H. Martin: Plane rotational flows.}

Taking the stream function $\psi$ and pressure $p$ for independent variables, the determination of the steady, rotational flow of a nonviscous fluid subject to no external forces reduces to the integration of the quasi-linear partial differential equation $(\partial / \partial \psi)\left(\left(q_{p p}-q \theta_{p}^{2}\right) / \theta_{\psi}\right)+q \theta_{p p}+2 q_{p} \theta_{p}=0$, where $\theta$ denotes the angle of inclination of the 
velocity vector and $q=q(\psi, p)$, the speed of flow is determined by Bernoulli's theorem when the equation of state for the fluid is given, and the variation of entropy and energy from stream line to stream line are prescribed. Flows for which the stream lines are isobars or for which $\theta=\theta(p)$ are excluded from the analysis and are treated separately. In the latter case a generalization of Prandtl-Meyer flows to rotational flows is obtained. The case $\theta=\theta(p)$ leads to a treatment of those flows in which the stream lines are all straight lines. (Received February 24, 1949.)

\section{1t. Eric Reissner: Note on the problem of twisting of a circular ring sector.}

It is shown that the partial differential equation for this problem (which is discussed on pp. 355-361 in Theory of elasticity by S. Timoshenko) can be integrated in closed form when the cross section of the ring sector is a hollow section with thin walls. The results obtained correspond in nature to those of R. Bredt for St. Venant torsion of cylindrical rods. (Received February 14, 1949.)

\section{GEOMETRY}

312. H. V. Craig and B. B. Townsend: On certain extended components of connection.

The derivatives with respect to the curve parameter of tensors whose first intrinsic derivatives vanish are shown to be linear in the tensors and have the extended components of connection, $L_{d}^{\alpha a}$ and $L_{\alpha a}^{o}$, for coefficients. These relations are applied to the problem of obtaining higher order intrinsic derivatives of absolute tensors of all orders as complete or reduced range contractions of certain extensors with the extended components of connection. These results are used to investigate the reduced range and full range contractions of the extended components of connection, $L_{d}^{\alpha}$, $L_{\alpha a}, g_{d}^{\alpha_{a}}$, and $g_{\alpha a}^{o}$. The particular contraction, $g_{d}^{\alpha_{a}} L_{\alpha a}^{0}$, for $M=2$, yields a tensor which may be used as a characterization of the flatness of a space. (Received February 23, 1949.)

\section{F. A. Ficken: Elementary reduction of a matrix to Jordan} normal form.

If $\lambda$ is an eigenvalue of $M$, and $L=M-\lambda I$, one can distinguish, among the vectors annulled by $L$, those which are and those which are not in the column space $C_{L}$ of $L$. If $L Z_{1}=0$, with $Z_{1}$ in $C_{L}$, find $Z_{2}$ such that $L Z_{2}=Z_{1}$, and continue until $L Z_{k+1}=Z_{k}$ with $Z_{k+1}$ not in $C_{L}$. Do this for each vector of a basis for the intersection of $C_{L}$ with the nullspace of $L$. All vectors so produced, along with those eigenvectors not in $C_{L}$, span the root space corresponding to $\lambda$. In this way obtain a basis for the root space for each eigenvalue $\lambda$. In the resulting basis for the whole space, $M$ will be in Jordan normal form. It is assumed that the eigenvalues are known; but successive $Z_{k}$ are obtained, for a given $\lambda$, merely by introducing a succession of right members for the same system of linear equations; neither the adjoint nor powers of $M$ are needed. Justification of the process is based on the Hamilton-Cayley theorem and an analysis of several spaces associated with $M$; elementary divisors are not used. (Received February 17, 1949.) ities.

314. G. B. Huff: Cremona's equations and the properness inequal- 
A solution $x=\left\{x_{0} ; x_{1}, x_{2}, \cdots, x_{n}\right\}$ of Cremona's equations, (1) $x_{0}^{2}-x_{1}^{2}-x_{2}^{2}-\cdots$ $-x_{n}^{2}=d+p-1,3 x_{0}-x_{1}-x_{2}-\cdots-x_{n}=d-p+1$, is said to be proper if there exists a linear system of plane curves of genus $p$, dimension $d$, order $x_{0}$, and having multiplicites $x_{1}, x_{2}, \cdots, x_{n}$ at a set of $n$ general points. A proper solution $x$ of (1) also satisfies the properness inequalities, (2) $y_{0} x_{0}-y_{1} x_{1}-y_{2} x_{2}-\cdots-y_{n} x_{n} \geqq 0$, where $y$ ranges over the finite number of characteristics of Bertini $L$-curves of $y_{0}<x_{0}$. By means of lemmas concerning minimal order solutions of (1) and (2), the following theorems are proved. I. Any solution of (1) satisfying the inequalities (2) is proper for $p=0$ and $d \geqq 0$, and there are proper solutions for each $d \geqq 0$. II. Any solution of (1) satisfying the inequalities (2) for $p=1, d \geqq 1$ is proper; and there exists a proper solution for $1 \leqq d \leqq 9$. For $d>9$, there are no proper solutions. (Received February 16, 1949.)

\section{Jack Levine: Field of parallel vectors in conformally flat spaces.}

If $C_{n}(p, q)$ denotes a conformally flat space admitting $p$ non-null and $q$ null fields of parallel vectors, it is shown that the only possibilities are $C_{n}(1,0)$ and $C_{n}(0,1)$. The $C_{n}(1,0)$ is a symmetric Cartan space of class one, and both types are spaces $K_{n}$ considered by Ruse in which $R_{h i j k_{1} m}=t_{, m} R_{h i j k}$, J. London Math. Soc. vol. 21 (1946) pp. 243-247. Invariant characterizations of a $C_{n}(1,0)$ and $C_{n}(0,1)$ are also obtained. Canonical forms of the type $u^{-2} \sum e_{i}\left(d x^{i}\right)^{2}$ are derived for the metrics of the spaces. The theory of product-spaces as developed by Ficken, Ann. of Math. (2) vol. 40 (1939) pp. 892-913, is used to obtain some of the results. (Received February 4, 1949.)

\section{B. E. Mitchell: A real representation of the imaginary line.}

The Laguerre representation of the imaginary point by an ordered pair of real points readily lends itself to construction in the complex plane. Such construction, however, cannot proceed far nor long, however, without a corresponding dual representation of the imaginary line. Furthermore, since the complex line and the complex point are dual elements in the complex plane, when they are united in position their representations should be organically united as Study has suggested. The ideal would be that if the point and the line are united in position, then their respective representations should also be united in position. This ideal is achieved, however, only when the line has a real trace and the point is real and lies on the trace. There is, nevertheless, a strong organic union in the general case. The real representation of the imaginary line is revealed in the discovery of a certain Gudermannian equivalent of the imaginary angle of the complex line and its conjugate. (Received April 2,1949.)

\section{7t. J. M. Thomas: Nomographic disjunction.}

The constants are from a commutative field. The variable domain consists of the triples $(x, y, z)$ obtained as each of the variables independently assumes the values in a corresponding given set. Each function assumes for each triple in the variable domain a single value from the field of constants. A disjoint is a determinant of order three with the following properties: the elements on the $i$ th row are functions of only the $i$ th one of the variables $x, y, z$; at least two of the functions in each row are linearly independent (with respect to the field of constants); and the algebraic complements of the three functions on each row are linearly independent. A function which equals a disjoint is a nomographic function. A function $F$ being given, the author develops an algebraic process (disjunction) for finding a disjoint $D$ which must equal $F$ if $F$ has a disjoint. The problem of finding whether $F$ is nomographic is thereby reduced to examining whether the single equation $F=D$ is an identity in $(x, y, z)$. (Received February 23, 1949.) 


\section{Statistics AND Probability}

318. Isadore Blumen: The effect of serial correlation on the distributions of certain statistics.

In obtaining the distributions of many commonly used statistics the assumption is made that the observations are independent. However, in time series problems, in agricultural experiments, and in other applications, it is frequently more reasonable to assume that the observations have a correlation depending on the "distance" between them. The exact probability distributions of certain statistics are obtained, as well as useful approximations, when it is assumed that the observations are sets of normally distributed variables with circular serial and lag correlations. (Received February 18, 1949.)

\section{A. C. Cohen: Estimating the mean and variance of truncated normal distribution.}

In certain types of scientific experiments it sometimes happens that the technique of measurement has a known limit beyond which the variate cannot be measured but the number of observations falling beyond this limit in any given sample can be counted. In this paper the method of "maximum likelihood" has been employed to obtain estimates of the mean and variance of normally distributed populations when the sample data are thus restricted. Variances of these estimates for large values of $n$ are also obtained. (Received February 17, 1949.)

320. G. E. Nicholson: On the estimation of the parameters of $a$ normal p-variate distribution from an incomplete sample.

In statistical investigations involving multicharacter observations on individuals it sometimes happens that certain observations on some of the characters are missing. The problem of utilizing these incomplete measurements in the estimation of the parameters of the population is treated when it is assumed that the underlying population is multivariate normal. The estimates are obtained in terms of familiar statistical quantities and certain conclusions pertaining to statistical prediction are obtained. (Received February 18, 1949.)

321. H. E. Robbins and E. J. G. Pitman: Application to the method of mixtures to quadratic forms in normal variates.

If $F_{n}(x)$ are distribution functions and if $c_{n}$ are constants such that $c_{n} \geqq 0$ and $\sum c_{n}=1$, then $F(x)=\sum c_{n} F_{n}(x)$ is a distribution function, called a mixture. Some useful properties of mixtures are derived and the results are applied to the distribution of a positive quadratic form in normal variates and of the ratio of two such forms. The solutions are given respectively as mixtures of gamma and of beta distributions, with an upper bound to the error involved in replacing the series by finite sums. (Received February 18, 1949.)

\section{TOPOLOGY}

322. I. C. Gentry: On extending a homeomorphism between subsets of 2-dimensional orientable manifolds. Preliminary report.

Let $M_{i}(i=1,2)$ denote two 2-dimensional orientable manifolds of genus $n$. Let $K_{i} \subset M_{i}$ be a Peano continuum. Let $\phi\left(K_{1}\right)=K_{2}$ be a homeomorphism defined over 
$K_{1}$ onto $K_{2}$. It is proved that in order that this homeomorphism shall be extendable to a homeomorphism $\phi^{\prime}, \phi^{\prime}\left(M_{1}\right)=M_{2}$, it is necessary and sufficient that the following conditions hold: (1) $\epsilon\left\{\phi\left(\gamma_{1}\right)\right\}=\epsilon\left(\gamma_{1}\right)$ for every index curve $\gamma_{1}$ of $K_{1}$ where $\epsilon\left(\gamma_{1}\right)$ is the signature of $\gamma_{1}$. (2) If $L_{1}$ is a linear graph in $K_{1}$ composed of a finite number of simple closed curves $\leqq$ genus of $M_{1}$ and $M_{1}-L_{1}$ is the sum of components $\sigma_{j}^{1}, j=1,2, \cdots, m$, then $M_{2}-\phi\left(L_{1}\right)$ is the sum of components $\sigma_{j}^{2}, j=1,2, \cdots, m$, such that (a) if $x \in K_{1}$ $\cdot \sigma_{j}^{1}$, then $\phi(x) \in \sigma_{j}^{2}$ and (b) for each $j, \overline{\sigma_{i}^{1}}$ is homeomorphic to $\overline{\sigma_{j}^{2}}$. This is a generalization of similar theorems by Adkisson and MacLane for the sphere and Knobelauch for the torus. (Received February 18, 1949.)

\section{O. G. Harrold: Euclidean domains with uniformly abelian} local fundamental groups. II.

In the first paper with this title the author showed that if the complementary subset to a topological $k$-cell $C$ in the $n$-dimensional spherical space $S^{n}$ has uniformly abelian local fundamental groups, then the group of $S^{n}-C$ is trivial. In this note the same hypothesis is placed on $S^{n}-C$ and the question is considered of what conditions may be placed on a subset $T \subset C$ in order that $S^{n}-T$ have a trivial group. If dimension $C=n$, the problem reduces essentially to one of the first paper. If dimension $C<n-1$, it is sufficient that $T$ be closed. If dimension $C=n-1$, it is sufficient that $T$ be an absolute retract. The main obstacle to be overcome when $\operatorname{dim} C=n-1$ is that of establishing a sense of two sides for an arbitrary closed, connected subset in the interior of $C$ relative to $S^{n}-C$. (Received February 16,1949.)

324t. Samuel Kaplan: Extensions of the Pontrjagin duality. II. Direct and inverse sequences.

Let $\left\{H_{\lambda}\right\}$ be a direct system of topological groups, that is, the index set is directed (for every pair $\lambda, \mu$, there is a $\nu$ such that $\lambda<\nu, \mu<\nu$ ) and for each pair $\lambda<\mu$ there is a continuous homomorphism $p_{\lambda \mu}: H_{\lambda} \rightarrow H_{\mu}$ such that if $\lambda<\mu<\nu$, then $p_{\lambda_{\nu}}=p_{\mu \nu} p_{\lambda \mu}$. The direct limit $\mathfrak{S}$ of the system $\left\{H_{\lambda}\right\}$ is defined as follows: Let $H=P^{w} H_{\lambda}$ topologized as in Kaplan, Extensions of the Pontrjagin duality. I, Duke Math. J. vol. 15 (1948); let $H_{0}$ denote the subgroup of $H$ generated by all elements of the form $h_{\lambda}-p_{\lambda \mu} h_{\lambda}, h_{\lambda} \in H_{\lambda}$, $\lambda$ running through the entire index set $\{\lambda\}$; then $\mathfrak{S}=H / \bar{H}_{0}$. It is proved that if $\left\{G_{n}\right\}(n=1,2, \cdots)$ is an inverse sequence of locally compact groups and $\left\{H_{n}\right\}$ the "dual" direct sequence of the corresponding character groups, then the inverse limit of the $G_{n}$ 's and the direct limit of the $H_{n}$ 's are character groups of each other. (Received February 9, 1949.)

\section{J. W. Keesee: Finitely-valued cohomology groups.}

Following the notation of Spanier (Ann. of Math. (1948) pp. 405-427) define $F^{p}(X, A)$ to be the subgroup of $\phi^{p}(X, A)$ consisting of those $p$-functions that assume only a finite number of elements of the coefficient group as values. $H_{F}^{p}(X, A)$ is then the cohomology group of $X \bmod A$ based on elements of $F^{p}(X, A)$. A homomorphism $\tau$ on $H_{F}^{p}(X, A)$ into $H^{p}(X, A)$ is exhibited, and it is proved that if $X$ is compact Hausdorff and $A$ is closed, then $\tau$ is an isomorphism onto. (Received February 16, 1949.) 